\title{
Social Delay Tolerant Protocol for Safety Services in Vehicular Networks
}

\author{
Huong Nguyen-Minh ${ }^{1}$, Abderrahim Benslimane ${ }^{1}$, Milena Radenkovic ${ }^{2}$ \\ ${ }^{1}$ Laboratoire Informatique d'Avignon, University of Avignon, Avignon, France \\ ${ }^{2}$ School of Computer Science, University of Nottingham, Jubilee Campus, Wollaton Road, Nottingham NG8 1BB, UK
}

\begin{abstract}
Vehicular networks have attracted attention for recent years due to their various and emerging applications supporting secure and convenient driving. Regarding specific features of vehicular networks, we propose a new Social-aware Vehicular DTN protocol (SocVe) respectively for a type of safety applications such as emergency support services. We evaluate our protocol in short contact and intermittent connection scenarios extracting from mobility data set in Hanoi city. We conduct comparative performance evaluation of SocVe in multiple scenarios with different destination centralities against a geographical protocol.
\end{abstract}

Keywords-Vehicular networks; social DTN; routing; safety applications.

\section{INTRODUCTION}

VANETs are well-known concept and are being deployed in increasing new applications. One kind of such applications in new environment that aims to improve transportation systems is emergency service in vehicular networks. It helps predict, avoid accidents or any safety-related incidents occurring in the road and recover after as well. However, the nature of wireless communication and vehicular environment including dynamic mobility, strong fading that leads to disconnection, and lack of centralization creates challenges for supporting these applications. Two of them are dissemination to vicinity area and communication with particular destinations.

Several dissemination and routing methods are proposed specially for vehicular networks. Majority of them, [1]-[4] [9], are location-based protocols based on geographic knowledge to disseminate and forward the packet. Vehicles commonly move from dense zones to completely sparse areas [8] so that protocols have to deal with both congestion and fragmentation thus intelligent delay tolerant protocol is proposed. Some protocols combine delay tolerant network (DTN) characteristics and geographic methods MDDV[5], CarNet[1], GeoDTN+Nav[3], VADD[4] and historical mobility patternbased protocol [2] for routing a packet from a source node to a destination node or a region. More recent works consider better network condition adapted metrics compared to geographical graphs, these are social graphs [6] [11] in Mobile Adhoc Network (MANET). These concepts are extended to be used in a high dynamic network, Vehicular Adhoc Network
(VANET)[7]. However, none of the related works investigate their protocols specifically for safety services which are important emerging applications in vehicular networks. Besides, there is a lack of comprehensive studies of social graphs with consideration of challenges coming from wireless adhoc vehicular environment (WAVE) characteristics.

Concerning features of WAVE such as short connection duration, transmission errors due to collision and bad link condition in wireless medium, in this paper, our main contributions are in two folds: we propose SocVe, a Socialaware Vehicular DTN protocol for communication between general vehicles and special entities that are emergency supporting services such as mobile police cars, fire trucks, ambulances or static agency; we study our protocols in a new mobility dataset of Hanoi city concerning about mentioned vehicular traits. These strike properties of vehicular environment must not be ignored in studies of safety application, especially, emergency incidents commonly come up with bad condition connectivity. Additionally, in emergency cases, vehicles likely do not travel as their intended pattern or historical paths. Besides, road side unit may not always available for communication in case of local nature disaster or they are targets of attacks. We operate our protocols without assumption of any predefined or historical mobility pattern and the availability of road side units.

The remainder of this paper is organized as following: Section II briefly reviews related works; section III describes emergency scenarios and our proposed protocols; the experiment setup is elaborated in section IV; simulation results are reported in section V; the paper is concluded in section VI.

\section{RELATED WORKS}

In recent years, there has been extensive work on social aware DTNs and opportunistic networks [1]-[4] while social graphs analytics have still not been studied sufficiently in vehicular ad hoc networks domain. Current disruption aware vehicular networks typically utilize DTN metrics such as trajectory, receive rate [3], and delay [4]. This paper aims to explore how integration of more complex contact analytics (social metrics) can be beneficial in VANET especially for safety applications.

CarNet[1] proposed a scalable location service that helps to forward packet geographically. MDDV[5] generates 
forwarding trajectory based on neighbor's mobility knowledge. In GeoDTN+Nav[3], nodes operate in 2 modes: perimeter mode and DTN mode utilizing geographical metrics and connection condition metrics. However, it works under an assumption that all nodes share their trajectory information which may relate to privacy problem. VADD[7] chooses forwarding path not only based on geographic distance, velocity but also the delay to destination of this path. Similarly, historical mobility pattern protocol [2] selects forwarding path based on knowledge of historical paths which is exchanged among neighbor. They require an installation of preloaded digital map which provides traffic statistic and predefined vehicles' trips.

Above protocols work under assumption of map knowledge and historical mobility pattern and they do not consider the constraints of radio resources, i.e., exchanged information between neighbors should be very limited, especially in dense and congested areas. Different from them, we propose a protocol which works irrespectively the need of map knowledge. Additionally, resource constraints are taken into account by limiting the information exchanging in periodical messages between neighbors.

Fitting better to mobile networks in term of adapting to topology changes, social-aware DTN protocol have been proposed in MANET such as SimBetTs[6], and additionally congestion-aware like CafRep[11]. [7] proposes social based forwarding protocol in vehicular network with the support of roadside units. As social graphs reflect node's ability of encountering other neighbors, utilizing social relation allows packets forwarded to popular area where they have more chance to find clues of their destinations.

We consider also social-aware protocol for VANET, however, our assumptions, our objective and identified scenarios are different. We focus on safety applications in scenarios of emergency. Radio transmission is modeled with its radio environment's nature such as propagation loss, distortion and collisions.

\section{PROPOSED PROTOCOL}

Beside hazadous warning application, there are other safety applications that is less urgent but important safety related services such as emergency services following an incident to update the status, request for search and rescure service, or support accident preventing, e.g. [13] introduce an artificial intelligence methodology that analyses current traffic flow data obtained from road sensors and makes a prediction regarding accidents. The communication between traffic controller and relevant vehicles allows an intermediate traffic adjustment that may reduce the risk of accidents happen. In order to support this kind of safety applications, we propose a scenario which describes the communication between vehicles to one or more predefined important nodes in the network denoted by the term "Point of Interest". These nodes can be mobile or static. This type of safety application requires high reliability while accepts looser delay constraint compared to hazardous safety-related application. Dealing with high dynamic topology in vehicular networks, we search for network characteristics which vary more slowly but still reflect the instant topology on the other hand. Several researchs in MANET found that there is social relationship existing amongs nodes and it can reflect the capability of encounter other members in the network. [15] discussed social properties in vehicular networks. Their analysis show that some concepts of social networks are hold for VANET. Social graphs can be good metrics to determine data carriers. Addressing to the application's requirement and feature of vehicular network such as fragmentation and intermittent connection, we propose using specified centrality metrics in our Social-aware intelligent Vehicular DTN protocol (SocVe).

Social graph can be quatified by how popular a given node is within network, called centrality and how strength the relationship is, called tie strength. According to Freeman and Elizabeth [6], centrality is defined based on the number of direct links that involve a given node called node degree, the mutual knowledge among its neighbors called betweenness and the number of reachable nodes in the network namely closeness. Because of the mobility, links between nodes are time-varying, tie strength graph is envisioned. It provides the information of how frequent and recent a given node meets its neighbors. The more frequent and recent neighbor is, the stronger relationships they have. Besides, we can predict likely connection by using information from the past, for example, if two nodes have high number of common neighbors, their future encounter likely occurs. They called it the similarity. In this work, we utilize node degree, betweenness, tie strength and similarity due to their less controled data exchanged but rich information obtained.

The design of SocVe including SocVe beacon format, SocVe data header and connectivity graph analytics are described in following subsections.

\section{Beacon and data header design}

Beacons are periodic messages exchanged among nodes which are within transmission range of each other. The use of beacons aims to announce the presence of a node and its social graphs. We design the beacon with a concern of optimizing the size of beacons in order to reduce collision among beacons or between beacons and data packets in radio medium.

\begin{tabular}{|c|c|c|c|c|c|}
\hline $\begin{array}{c}\text { Type } \\
=0 \\
(1 \mathrm{bit})\end{array}$ & $\begin{array}{c}\text { Header } \\
\text { Length } \\
(7 \mathrm{bits})\end{array}$ & $\begin{array}{c}\text { PoI List } \\
\text { Length } \\
\text { (1Bytes) }\end{array}$ & $\begin{array}{c}\text { Contact List } \\
\text { (varied size) }\end{array}$ & $\begin{array}{c}\text { Betweenness } \\
\text { (8Bytes) }\end{array}$ & $\begin{array}{c}\text { PoI List } \\
\text { (varied } \\
\text { size })\end{array}$ \\
\hline
\end{tabular}

Fig.2. a. Beacon format

\begin{tabular}{|c|c|c|}
\hline $\begin{array}{c}\text { Type }=1 \\
(1 \mathrm{bit})\end{array}$ & $\begin{array}{c}\text { Header Length }=2 \\
\text { (7bits) }\end{array}$ & $\begin{array}{c}\text { Destination ID } \\
\text { (2Bytes) }\end{array}$ \\
\hline
\end{tabular}

Fig.2. b. Data header format 
A beacon format shown in Fig.2a comprises a type field keep as 0 for beacon and 1 for data header, a header length field, PoI List Length that shows the number of encountered PoI, Contact List field includes the list of encountered neighbors, Betweenness field and PoI List includes the list of encountered PoI.

PoI List is composed by a list of PoI contact. PoI contact is a structure of PoI ID, tiestrength and similarity of the given node to encountered PoI. The size of PoI List and Contact List vary depending on social metrics of given node.

A SocVe data header described in Fig.2b is added to data packet in order to reserve destination ID during the forwarding process.

\section{Complex Connectivity Graph Analystics}

Based on exchanged beacons, vehicles calculate their social graphs in term of centrality, betweenness, and its tie strength and similarity to $\mathrm{PoI}$ if any encounter.

\section{- Centrality:}

Centrality of a vehicle includes degree - the number of its encounters during predefined time, and betweenness - the existing indirect links between each pair of its neighbors. Degree is computed as total number of direct links to given vehicle $n_{0}$.

$$
C_{D}\left(n_{0}\right)=\sum_{k=1}^{N} a\left(n_{0}, n_{k}\right)
$$

Betweenness can be defined as in [6] by building and processing adjacency matrix. The adjacency matrix is updated once a new beacon is received.

- Tie Strength

Frequency is referred by the number of time that a given vehicle encounters a PoI. Frequency graph of vehicle $n$ to PoI $p$ is calculated as below equation:

$$
F I_{n}(p)=\frac{f(p)}{F(n)-f(p)}
$$

on which $f(p)$ is the number of PoI $p$ encountered time, $F(n)$ is the total time that vehicle $n$ has encountered from the beginning of the simulation.

Recency is defined as how recently vehicle $n$ last met a PoI. It is computed as following equation:

$$
\operatorname{Rec}_{n}(p)=\frac{\operatorname{rec}(p)}{T(n)-\operatorname{rec}(p)}
$$

- Tie predictor

Once a vehicle encounters POI, it computes the similarity to the POI and update. More specifically, the similarity is defined as number of not only common direct neighbors but also indirect encounters. The higher number of common neighbors, the higher probability that a given vehicle moves regularly to across this PoI. To account for more synchronous communication which is appropriated for DTN scenario we use similarity as a core metric for calculating tie prediction.

\section{- Choosing carrier}

The decision of forwarding the stored packet is made based on the utility calculation whenever a source or a carrier detects a new neighbor. If the new neighbor has a higher total utility compared to the given vehicle, the packet will be forwarded. The utilities is computed by pair-wise comparison.

$$
\begin{gathered}
\operatorname{DegUtil}_{n}(m)=\frac{C_{D}(m)}{C_{D}(m)+C_{D}(n)} \\
\operatorname{TSUtil}_{n}(m)=\frac{T S_{m}(p)}{T S_{m}(p)+T S_{n}(p)} \\
\operatorname{SimUtil}_{n}(m)=\frac{\operatorname{Sim}_{m}(p)}{\operatorname{Sim}_{m}(p)+\operatorname{Sim}_{n}(p)} \\
\operatorname{SocVeUtil}_{n}(m)=\sum u_{n}(m)
\end{gathered}
$$

Vehicle $n$ decides next carriers based on total utility SocVeUtil $(m)$ calculation in (7). Respectively to selected connectivity graphs in our protocol, utilities $u_{n}(m)$ for vehicle $m$ are normalized by graphs of the given vehicle $n$ : degree utility $\operatorname{DegUti}_{n}(m)$, tie strength utility to $\operatorname{TSUtil}_{n}(m)$, similarity utility to PoI $p \quad \operatorname{SimUtil}_{n}(m)$ in (4)-(6). For simple, the total utility is the sum of all non-weighted social graphs utilities.

Packets are stored by carriers within duration of time namely store duration $D_{S}$. During the store duration, whenever a carrier detects a better one, it forwards the packet to that new carrier. Due to the highly dynamic topology characteristic of vehicular network, connection duration and isolated duration of vehicles has various value and distribution. Some vehicle has higher centrality has longer connection duration and likely in connected status than isolated status. On the other hand, the relation between store duration and connectivity exists. If $D_{S}$ is too short, all stored packet are likely neglected before carriers have a chance of forwarding packets, then DTN protocol does not work. Otherwise, $D_{S}$ is too long, high centrality vehicles which have a lot of neighbor overwhelmingly forward packets. It probably floods the network. Therefore, there should be a consideration of suitable value for $D_{S}$ regarding to connectivity of each node in the network. In early experiments, we choose a fixed value of $D_{S}$ of 300 seconds.

\section{EXPERIMENT SETUP}

In this paper, we would like to study a new metropolitan city dataset - Hanoi city where old French style transport system and modern roads coexist. This feature of Hanoi's roads system leads to a big diversity of road size and respectively various network density. In the old quarter, roads 
have only one lane while other parts in the map present cross where several four-laned roads encounter.

We evaluate our SocVe in NS-3. We consider a region of $5000 \mathrm{mx} 10000 \mathrm{~m}$ extracted from the map of urban districts in Hanoi city by SUMO mobility simulator.

\section{City map}

SUMO exported map in Fig.3b shows two bridges crossing the Red River, urban streets and a belt highway in blue color. City streets allow speed up to $40 \mathrm{~km} / \mathrm{h}$ while the highway requires the speed ranging from $70 \mathrm{~km} / \mathrm{h}$ to $110 \mathrm{~km} / \mathrm{h}$.

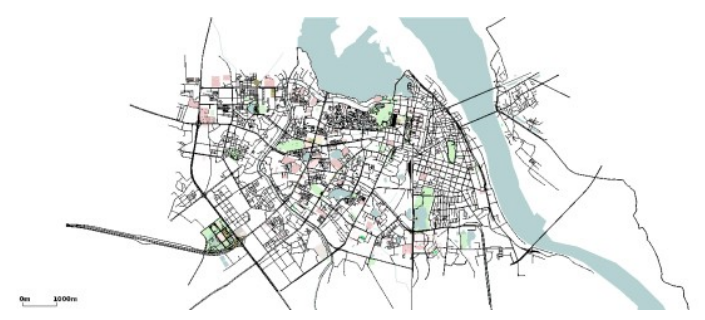

Fig.3. Extracted map

In order to set up the simulation, we use Simulator of Urban Mobility (SUMO) to generate nodes' mobility and NS-3 as a communication simulator. Nodes move in the network according to the mobility model extracted from SUMO which respects properties of roads in the city map. Nodes obey the natural regulation of transportation such as reasonable acceleration, distance between cars, traffic lights and speed limit. For communication protocol, we use NS-3 on which SocVe protocol is implemented and also lower layers are simulated. Specifically, MAC and physical layers are described by standard IEEE $802.11 \mathrm{p}$ with a bandwidth of $10 \mathrm{Mhz}$ and Nakagami-m propagation loss model which is suggested for Dedicated Short Range Comunication studies[10]. The combination of SUMO and NS-3 allows us to evaluate our protocols in a specific real city where road properties and respective traffic are closely and visually abstracted.

We evaluate SocVe with regard to the popularity of the PoIs. Depending on position, PoIs should have different level of centrality, as some PoIs in central area may encounter more nodes than the other in rural area. In order to study separately impact of different PoIs to performance of all network and of each individual PoI.

Three groups of common centrality PoIs are picked up in the map for simulation: high centrality (PoI ID 1, 2, 3), medium centrality (PoI ID 4, 5, 6), and low centrality (PoI ID $5,6,7)$. Referencing to the degree and betweenness calculation during the simulation, centrality of PoIs is presented in Fig.5. High centrality PoIs are located very central in the city such as next to the main train station or coach stations, along main streets and near to the Hoan Kiem Lake which marks the center of Hanoi, main post office and national bank. Medium centrality PoIs are chosen at about 4 to 5 kilometers around the centre where we find houses, schools and commercial center. Low centrality PoIs are close to the outskirt of the city.

Among cars, 3 random nodes are chosen as source nodes sending a group of $400 \mathrm{~B}$ safety messages to 3 PoIs every 200s. The experiment is repeated 3 times with the change of source nodes in order to avoid the case that sources are always high centrality or particular close neighbors to PoIs.

\section{Topology analysis}

Social networks analyze connectivity between nodes in graphs so they can also take into consideration physical density parameters. Topology can be modelized as a graph of contacts [14] such as degree centrality, betweeness centrality, similarity, recency etc - these are all different metrics that are telling us how important a node is and how well connected it is to the rest of the network. Using properties of dynamic complex graphs where individual nodes have these centralites for vehicular networks should be helpful to data communication and dissemination in these environments.

Every node sends periodically one beacon every $1 \mathrm{~s}$. A node is in contact with another if it receives at least one of his beacons within $3 \mathrm{~s}$. It is considered as isolated if it is not in contact with anyone.

In the context of mobility traces, we perform analysis of 150 nodes-Hanoi data set, 134 nodes-Rome traces [16] and 100 nodes-San Francisco Cab traces [17]. Hanoi scenario shows some strike differences. Fig. 4 performs comparison of

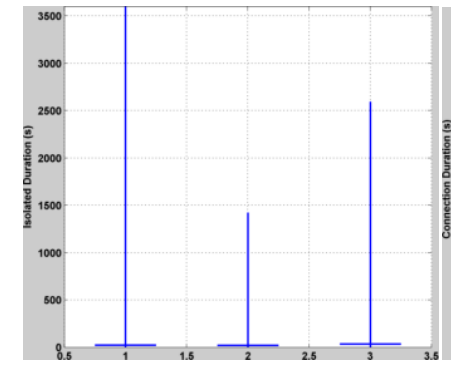

(a)

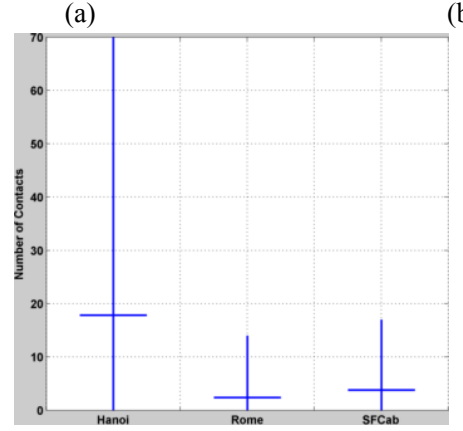

(c)

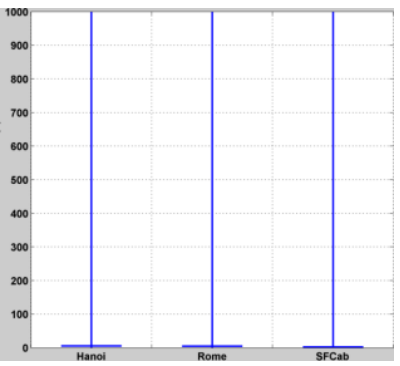

(b)
Fig.4: a. Isolation duration; b. Connection duration; c. Number of contacts

traces. Fig.4a and Fig.4b show isolation duration and connection duration. Hanoi has mean isolated duration of 
24.1s, maximum of $3596 \mathrm{~s}$ which is higher than $21.5 \mathrm{~s}$ in Rome data sets. On the other hand, Hanoi scenario's connection duration has mean of $5.74 \mathrm{~s}$, and maximum of $3600 \mathrm{~s}$ which is similar to in the other two data sets. It is significant high compared to transmission duration of a safety message which in milliseconds.

Fig.4c shows a diverse density in the network in Hanoi scenario. Although the mean number of contacts in Hanoi of 17.8 is much higher than the two others 2.4 and 3.81 respectively, this number varies a lot around the mean value, from 0 to 70 . It reflects a high dynamic topology where a given node likely moves from a very sparse region to a very dense one. Moreover, in term of radio medium, it shows a fluctuation in connecting among nodes in network, i.e, even nodes are close but they probably disconnected temporally due to wireless transmission collisions.

As our objectives are safety applications in vehicular networks, we focus on the characteristic of Wireles Adhoc Vehicular Environment (WAVE) which includes short connection and high affect of radio environment. Above features of Hanoi scenario match to our objectives.

\section{SimUlation RESUlts}

The simulation is set up with 120 cars entering the networks during first $100 \mathrm{~s}$ and 5 bus lines which have buses depart every 300 s. The simulation is executed in an hour, during that time the total number of nodes presenting in the network is 153 nodes including 3 PoIs, 30 periodic buses and 120 cars.

The performance of SocVe is shown in Fig.6. Respective Success Ratio and Delay to three groups of PoI are presented. The Success Ratio reach nearly $90 \%$ for scenario of high centrality PoIs. However, the delay shows its independence to the PoIs'centrality. Compared to higher centrality group, lower centrality group has longer delay, such as delay of a low centrality group is about $30 \%$ longer than its medium centrality group and delay of the medium centrality group is $40 \%$ longer than the high centrality group's.

Also we obtain an significant improvement of $80 \%-90 \%$ in success ratio of SocVe compared to scenario without using DTN protocol on which almost packets are lost, only $10 \%$ are received in high centrality PoI scenario due to the availability of connection in central area. This is shown in Fig.7 which proves that the protocol can deal with the fragmented network.

In Fig.7, we compare also SocVe to a geographical DTN routing protocol, Distance Aware Epidemic Routing (DAER) proposed for vehicular network which use geographic metrics instead of social graphs in [12]. They propose node's ability of storing and forwarding packets to a node that is closer to destinations. In order to limit the expanding rapidly stored and forwarded packets, carriers stop storing whenever they figure out that they are moving further from the destinations.

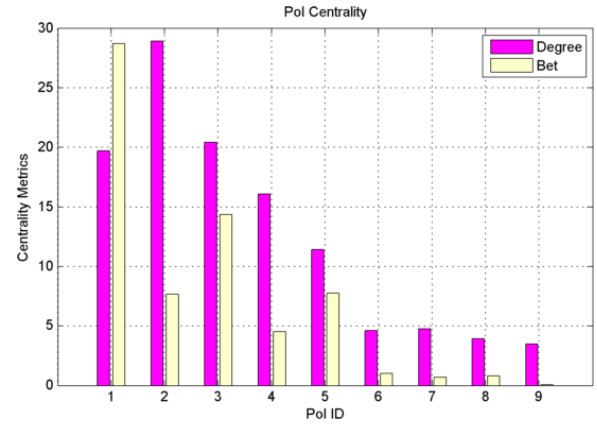

Fig.5. Centrality of Point of Interest (PoI)

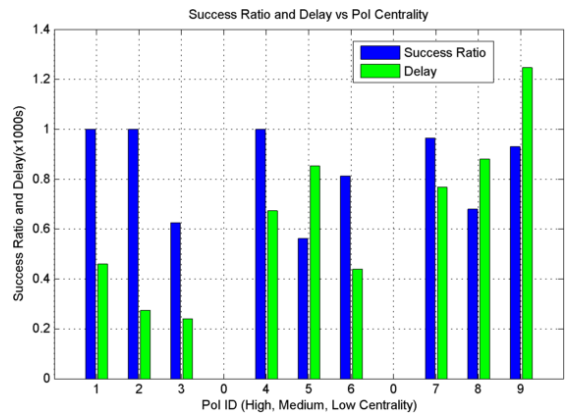

Fig.6. SocVe Success Ratio versus PoI Centrality

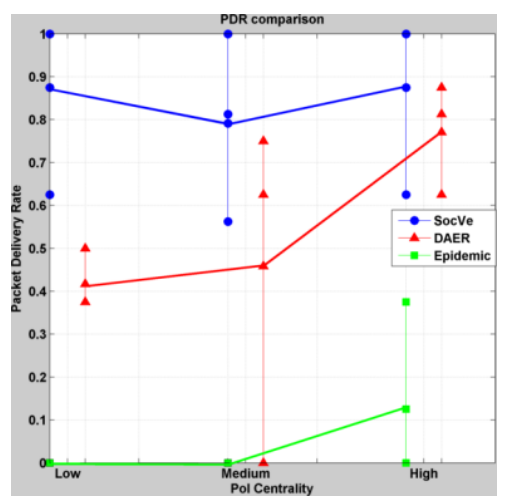

Fig.7. Success Ratio SocVe, DAER, Epidemic protocol

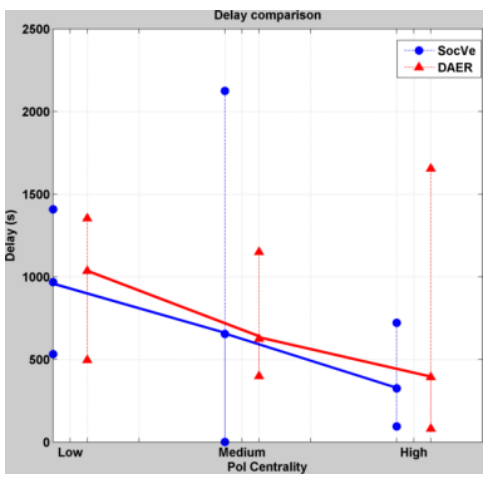

Fig. 8. Average Delay SocVe and DAER

SocVe shows a higher average success ratio of $40 \%$ compared to geographical DTN method for low and medium PoI groups. While both SocVe and DAER perform closely success ratio for high centrality PoIs, $88 \%$ and $78 \%$ respectively. Forwarding packet to a node that is closer to PoI 
help bring the packet closer to the PoI instantly. For high centrality PoI, many nodes have high probability to encounter PoI, many of them have approximate social heuristics thus social graphs do not outperform in this case; however without social- aware, packet doesn't have a chance to be brought to popular network region where it has more choices for next carriers and higher probability to meet the best carriers, therefore for lower centrality destination, social graphs seem to perform better.

Delays for received packets experiencing two protocols are shown in Fig.8. For low centrality PoIs, PoIs are rarely encountered, SocVe presents a average delay similarly to DAER. While in central scenario, the average delay of SocVe decreases significantly to 300 s and DAER delay reduces to 400s. From social aspect, not many nodes know rural PoIs thus it takes time for them to search someone in relation with the PoIs, it leads to a longer delay for low density PoIs.

In SocVe, carriers are decided base on complex connectivity graphs measured by number of contacts (centrality), their encounter frequency, encounter duration and number of common neighbors which vary gradually with respect to the movement of vehicles. Popular nodes are likely always in contact with many others. Consequently, these "well-known" nodes are prone to be carriers, so packets are routed to groups where potential next carriers are available with high probability no matter the dynamic change in physical network. Therefore, choosing social metrics allows SocVe to adapt to the fast changed topology; as a result, success ratio is improved.

\section{CONCLUSION}

Between two offered services in vehicular network, safety service is a recent and more challenged traffic type. Its variety maps to different emergency scenarios which have different constraints. Together with the outstanding physical characteristics of vehicular environment, it raises a demand of comprehensive study of routing protocol with regard to lower layer and respective mobility trace data sets. In this paper, we have introduced a social aware DTN routing protocol for less urgent but important safety messages. We map our protocols to a respective scenario and evaluate it in Hanoi map-based mobility dataset which instroduces short and intermittent connection amongs vehicles. We will continue the study for an optimal store duration which may support the protocol adapting to high diverse network topology.

\section{REFERENCES}

1. W. Chen, R. K. Guha, T. J. Kwon, J. Lee and YY.Hsu, "A survey and challenges in routing and data dissemination in vehicular ad hoc networks", Vehicular Electronics and Safety, 2008. ICVES 2008. IEEE International Conference on , vol., no., pp.328,333, 22-24 Sept. 2008.
2. S. Hosseininezhad, V.C.M. Leung, "Data Dissemination for Delay Tolerant Vehicular Networks: Using Historical Mobility Patterns", Proceedings of the third ACM international symposium on Design and analysis of intelligent vehicular networks and applications. ACM, 2013.

3. K.C. Lee and M. Gerla, "Opportunistic Vehicular Routing", Wireless Conference (EW), 2010 European. IEEE, 2010.

4. J.Zhao and G. Cao, "VADD: Vehicle-Assisted Data Delivery in Vehicular Ad-hoc Networks", Vehicular Technology, IEEE Transactions on 57.3 (2008): 1910-1922.

5. H. Wu and R. Fujimoto, R. Guensler and M. Hunter, "MDDV: A MobilityCentric Data Dissemination Algorithm for Vehicular Networks", Proceedings of the 1st ACM international workshop on Vehicular ad hoc networks. ACM, 2004.

6. E. M. Daly and M. Haahr, "Social Analysis for Information Flow in Disconnected Delay-Tolerant MANETs", Mobile Computing, IEEE Transactions on 8.5 (2009): 606-621.

7. R. Lu, X. Lin, X. Shen, "SPRING: A Social-based Privacy-preserving Packet Forwarding protocol in Vehicular Delay Tolerant Networks", INFOCOM, 2010 Proceedings IEEE, vol., no., pp.1,9, 14-19 March 2010.

8. G.S. Thakur , P. Hui, A. Helmy, "Modeling and Characterization of Vehicular Density at Scale", INFOCOM, 2013 Proceedings IEEE. IEEE, 2013.

9. A. Benslimane, "Optimized Dissemination of Alarm Messages in Vehicular Ad-hoc Networks (VANET)", High Speed Networks and Multimedia Communications Lecture Notes in Computer Science Volume 3079, 2004, pp 655-666.

10. H.Hartensteins, K.P.Labeteaux, "VANET: Vehicular Applications and Inter-networking Technologies", A John Willey and Sons, Ltd, Publication, pp 235-237.

11. M.Radenkovic, A.Grundy, "Efficient and adaptive congestion control for heterogeneous delay-tolerant networks", Ad Hoc Networks, Volume 10, Issue 7, September 2012, Pages 1322-1345, ISSN 1570-8705.

12. P.Luo, S. Jiao, H. Huang, W. Shu, M. Li, "Performance Evaluation of Vehicular DTN Routing under Realistic Mobility Models", Wireless Communications and Networking Conference, 2008. WCNC 2008. IEEE.

13. R. Jagannathan, S. Petrovic, G. Powell, M. Roberts, "Predicting Road Accidents Based on Current and Historical Spatio-temporal Traffic Flow Data", Computational Logistic, Lecture Notes in Computer Science Volume 8197, pp. 83-97, Springer-Verlag Berlin Heidelberg 2013.

14. A.Chaintreau, P.Hui, J.Crowcroft, C.Diot, R.Gass, J.Scott, "Impact of Human Mobility on Opportunistic Forwarding Algorithms", in Proc. INFOCOM, April 2006.

15. X.Liu, Z.Li, W.Li, S.Lu, X.Wang, D.Chen, "Exploring social properties in vehicular ad hoc networks", Proceedings of the Fourth Asia-Pacific

Symposium on Internetware (Internetware '12). ACM, New York, NY, USA, Article 24, 7 pages.

16. L.Bracciale, M.Bonola, P.Loreti, G.Bianchi, R. Amici, A.Rabuffi, “CRAWDAD data set roma/taxi (v. 2014-07-17)", Downloaded from http://crawdad.org/roma/taxi/, July 2014.

17. M. Piorkowski, N.Sarafijanovic-Djukic, M.Grossglauser, “CRAWDAD data set epfl/mobility (v. 2009-02-24)", Downloaded from http://crawdad.org/epfl/mobility/, Febuary 2009.
This work have been done in the frame of Perdiguer international mobility grant of University of Avignon, France; in collaboration with University of Nottingham, UK, 2014 